\title{
Intellectual disability, Wolff type
}

INSERM

\section{Source}

INSERM. (1999). Orphanet: an online rare disease and orphan drug data base. Intellectual disability, Wolff type. ORPHA:3080

Intellectual disability, Wolff type is a rare intellectual disability syndrome characterized by severe intellectual disability, characteristic facial features (low anterior hairline, upward slanting palpebral fissures, ocular hypertelorism, broad, bulbous nose, large ears with helix incompletely developed, thick lips, and microg nathia) and additional anomalies including peripheral joint contractures, delayed skeletal maturation, bilateral cleft lip and palate, strabismus, terminal hypoplasia of fingers, hypospadias, and bilateral inguinal hernias. 